\title{
Hábitos alimentares de Bryconamericus stramineus Eigenmann, 1908 (Characidae), em diferentes riachos da sub-bacia do Rio Guiraí, Mato Grosso do Sul, Brasil
}

\author{
Lucas Brandão-Gonçalves ${ }^{1,3}$, Sidnei Eduardo Lima-Junior ${ }^{2}$, Yzel Rondon Suarez ${ }^{2}$ \\ ${ }^{1}$ Unidade Universitária de Ivinhema, \\ Universidade Estadual de Mato Grosso do Sul - UEMS, \\ Av. Brasil, 679, Centro, CEP 79740-000, Ivinhema, MS, Brasil \\ ${ }^{2}$ Universidade Estadual de Mato Grosso do Sul-UEMS, \\ Unidade Universitária de Dourados, CINAM, \\ Rod. Dourados, Itahum, Km 12, Cidade Universitária, \\ CEP 79804-970, Dourados, MS, Brasil \\ ${ }^{3}$ Autor para correspondência: Lucas Brandão-Gonçalves, e-mail: lucasbrandaog@yahoo.com.br
}

BRANDÃO-GONÇALVES, L., LIMA-JUNIOR, S.E. \& SUAREZ, Y.R. 2009. Feeding habits of Bryconamericus stramineus Eigenmann, 1908 (Characidae), in different streams of Guiraí River Sub-Basin, Mato Grosso do Sul, Brazil. Biota Neotrop. 9(1): http://www.biotaneotropica.org.br/v9n1/en/abstract?article+bn03109012009.

\begin{abstract}
Feeding habits of Bryconamericus stramineus were described in different streams of Guiraí River Sub-basin in Mato Grosso do Sul State, Mid-West of Brazil. Fishes were sampled bi-monthly between October/06 and August/07 in seven streams and the samples were separated in two seasons: dry and wet. A total of 960 individuals were captured. B. stramineus showed an insectivore feeding habit, consuming seventeen food items. The feeding tactic of preys capture varied between the seasons and the locations. During the wet season the most important items were Hymenoptera, Diptera and Coleoptera, while Megaloptera and Diptera were the most important during dry season. In all analyzed streams, B. stramineus showed a diet basically composed by insects; the other items spent sometimes, corroborating the literature.
\end{abstract}

Keywords: diet, insectivory, characin.

BRANDÃO-GONÇALVES, L., LIMA-JUNIOR, S.E. \& SUAREZ, Y.R. 2009. Hábitos alimentares de Bryconamericus stramineus Eigenmann, 1908 (Characidae), em diferentes riachos da sub-bacia do rio Guiraí, Mato Grosso do Sul, Brasil. Biota Neotrop. 9(1): http://www.biotaneotropica.org.br/v9n1/pt/ abstract?article+bn03109012009.

Resumo: Os hábitos alimentares de Bryconamericus stramineus foram estudados em riachos da sub-bacia do rio Guiraí, no estado de Mato Grosso do Sul, centro-oeste do Brasil. As amostragens foram realizadas bimestralmente em sete riachos na bacia do rio Guiraí, Alto Rio Paraná, de outubro/2006 a agosto/2007. Foram capturados 960 exemplares. As amostragens foram divididas em estação chuvosa e estação seca. B. stramineus apresentou hábito alimentar insetívoro; a espécie utilizou 17 itens alimentares, sendo que a tática de consumo das presas variou entre as estações a as localidades. Durante a estação chuvosa nos estômagos prevaleceram as Ordens Hymenoptera, Diptera e Coleoptera, durante a seca predominaram as Ordens Megaloptera e Diptera. Em todos os riachos analisados, B. stramineus apresentou dieta basicamente composta por insetos, sendo os demais itens consumidos ocasionalmente, confirmando a literatura.

Palavras-chave: dieta, insetivoria, caracídeo. 


\section{Introdução}

A vasta riqueza de espécies de peixes em sistemas aquáticos tropicais levanta uma questão relevante a respeito de como os recursos disponíveis no ambiente são partilhados entre as espécies que compõem a comunidade (Lowe-McConnel 1999). Ao contrário dos organismos terrestres, para os quais a segregação de habitat é mais importante, para as comunidades aquáticas a segregação trófica tem se mostrado a mais expressiva (Ross 1986).

As diversas estratégias e táticas de forrageamento desenvolvidas pelos peixes possibilitam que eles façam uso dos mais diferentes recursos alimentares disponíveis nos ambientes aquáticos e em seus entornos. Wootton (1992) comenta que os peixes podem ocupar todos os níveis tróficos da cadeia alimentar. Portanto, o alimento consumido permite reconhecer dentro da ictiofauna grupos tróficos distintos e inferir sobre a sua estrutura, grau de importância dos diferentes níveis tróficos e inter-relações entre seus componentes. Investigações sobre o espectro alimentar de peixes, por meio de estudos de seus conteúdos estomacais, auxiliam na interpretação da dinâmica e ocupação de habitats por tais espécies.

A flexibilidade de hábito alimentar é uma característica adaptativa do comportamento animal, uma vez que ambientes naturais variam espacial e temporalmente. Assim sendo, variações sazonais normalmente ocorrem na alimentação desses animais em resposta à flutuação temporal dos fatores bióticos e abióticos que determinam a disponibilidade de recursos alimentares no ambiente (Lowe-McConnel 1999, Wooton 1992, Zavala-Camin 1996, Abelha et al. 2001). Além disso, as eventuais variações espaciais na dieta que as espécies podem apresentar ao se considerar locais geograficamente próximos (Wootton 1992, Hahn et al. 1997, Lima-Junior 2004) também devem ser consideradas, uma vez que contribuem na compreensão da dinâmica trófica da ictiofauna e sua relação com as características de cada ambiente.

Estudos sobre alimentação de peixes vêm se tornando cada vez mais numerosos devido à necessidade de dados para a formulação de modelos sobre a estrutura trófica de ecossistemas, além do entendimento dos mecanismos biológicos de interações inter-específicas, tais como predação e competição (Zavala-Camin 1996).

Com base nesse contexto, o objetivo deste trabalho foi caracterizar a dieta de Bryconamericus stramineus Eigenmann, $1908 \mathrm{em}$ riachos da sub-bacia do rio Guiraí - MS, atentando para o fato de que essa espécie pode apresentar variações sazonais ou espaciais em sua dieta.

\section{Materiais e Métodos}

As amostragens foram realizadas bimestralmente em sete riachos na bacia do rio Guiraí, Alto Rio Paraná, de outubro/2006 a agosto/2007 (Figura 1). Sete riachos foram selecionados de acordo com a posição na rede de drenagem e facilidade de deslocamento por

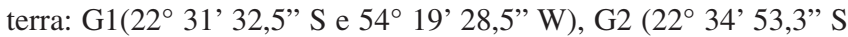
e $\left.54^{\circ} 19^{\prime} 42,1^{\prime \prime} \mathrm{W}\right), \mathrm{G} 3$ ( $22^{\circ} 36^{\prime} 12,3^{\prime \prime} \mathrm{S}$ e $\left.54^{\circ} 20^{\prime} 26,8^{\prime \prime} \mathrm{W}\right), \mathrm{G} 4$

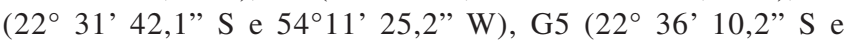

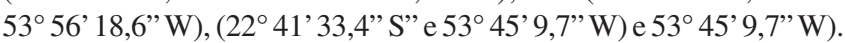
$\mathrm{O}$ ponto G3 (Figura $2 \mathrm{c}$ ) é cercado por uma área de pastagem e é o que apresenta o menor volume, com menos largura e profundidade, enquanto o ponto $\mathrm{G} 7$ (Figura $2 \mathrm{~g}$ ) é o mais volumoso, com $6,5 \mathrm{~m}$ de largura e $126 \mathrm{~cm}$ de profundidade média. O local com maior velocidade média da correnteza foi o G6 (Figura 2f) que apresenta uma mata densa em sue entorno, enquanto os pontos G1 (Figura 2a) e G7 foram os que apresentaram menor velocidade da correnteza, sendo que o G7 apresenta uma pequena área alagável em suas margens, com predomínio de Eichornia azurea e Echinodorus sp., com grande quantidade de matéria orgânica no sedimento, o ponto G2 (Figura 2b) é rodeado por gramíneas e apresenta o segundo maior volume de água com 5,2 $\mathrm{m}$ de largura e $73 \mathrm{~cm}$ de profundidade média, os locais G4 e G5 apresentam volume bem aproximado, ambos com $51 \mathrm{~cm}$ de profundidade média, 4,4 e 4,5 m de largura média respectivamente, mas diferem significativamente em termos de vegetação marginal, G5 (Figura 2e) apresenta mata ciliar enquanto G4 é cercado por gramíneas (Figura 2d). As comparações das demais características limnológicas estão listadas na Tabela 1.

As amostragens foram realizadas utilizando-se telas retangulares de $0,80 \times 1,20 \mathrm{~m}$ com malha de $2 \mathrm{~mm}$. Os exemplares coletados foram fixados, ainda em campo, em solução de formol $10 \%$ e transferidos posteriormente para uma solução aquosa de álcool etílico $70 \%$ (conservante).

Os indivíduos coletados nas unidades amostrais - definida como a amostragem de um dos córregos em um determinado mês - representadas por um mínimo de oito exemplares tiveram sua dieta analisada a partir dos métodos utilizados por Lima-Junior (2004), descritos a seguir.

Para inferência da dieta, o conteúdo estomacal de cada indivíduo foi retirado, pesado (em g, com aproximação de $0,01 \mathrm{~g}$ ) e posteriormente analisando sob estereomicroscópio (lupa). Após a identificação dos itens alimentares segundo a literatura especializada (Peterson1960, Needham \& Needham 1982, Barnes 1990), os dados obtidos em cada unidade amostral foram analisados a partir dos seguintes métodos:

Frequiência de ocorrência (F): é a porcentagem de estômagos, em relação ao total de estômagos com conteúdo em uma amostra, que apresenta uma determinada categoria alimentar. É calculada pela seguinte fórmula (Hyslop 1980):

$$
\mathrm{F}_{\mathrm{i}}=100\left(\mathrm{n}_{\mathrm{i}} / \mathrm{n}\right)
$$

sendo: $F_{i}$ : Freqüência de Ocorrência do item i na dieta dos indivíduos da amostra; $\mathrm{n}_{\mathrm{i}}$ : número de estômagos da amostra contendo o item $i$; e n: número total de estômagos com conteúdo na amostra.

Índice de análise volumétrica (V): esse índice indica a abundância relativa de determinado item alimentar na dieta dos animais de uma amostra. Para tanto, utiliza-se como referência a Massa Padrão, conceito correspondente à média aritmética das massas dos conteúdos estomacais de uma espécie, calculada com uma amostragem prévia e utilizada como um valor constante para todas as amostras da espécie em questão. Cada conteúdo estomacal recebe um total de pontos que é proporcional à razão de sua Massa Padrão, que recebe quatro pontos. Posteriormente, e com base em uma simples inspeção visual, os pontos totais atribuídos são divididos entre os itens alimentares de acordo com o volume relativo que estes ocupam no conteúdo do estômago.

O somatório de pontos recebidos por cada item alimentar dividido pelo número total de estômagos com conteúdo na amostra forneceu a média dos valores atribuídos para cada item alimentar. Posteriormente, essa média foi empregada no cálculo do Índice de Análise Volumétrica, feito a partir da seguinte fórmula (Lima-Junior \& Goitein 2001):

$$
\mathrm{V}_{\mathrm{i}}=25 \mathrm{M}_{\mathrm{i}}
$$

sendo: $\mathrm{V}_{\mathrm{i}}$ : Índice de Análise Volumétrica do item i na dieta dos indivíduos da amostra;25: constante e multiplicação para obtenção de um resultado percentual; e $\mathrm{M}_{\mathrm{i}}$ : média dos valores atribuídos para o item alimentar i na amostra;

Índice de importância dos itens alimentares (AI): esse índice indica a importância relativa de determinada categoria alimentar na dieta de uma amostra de indivíduos. É calculado da seguinte forma (Lima-Junior \& Goitein 2001): 


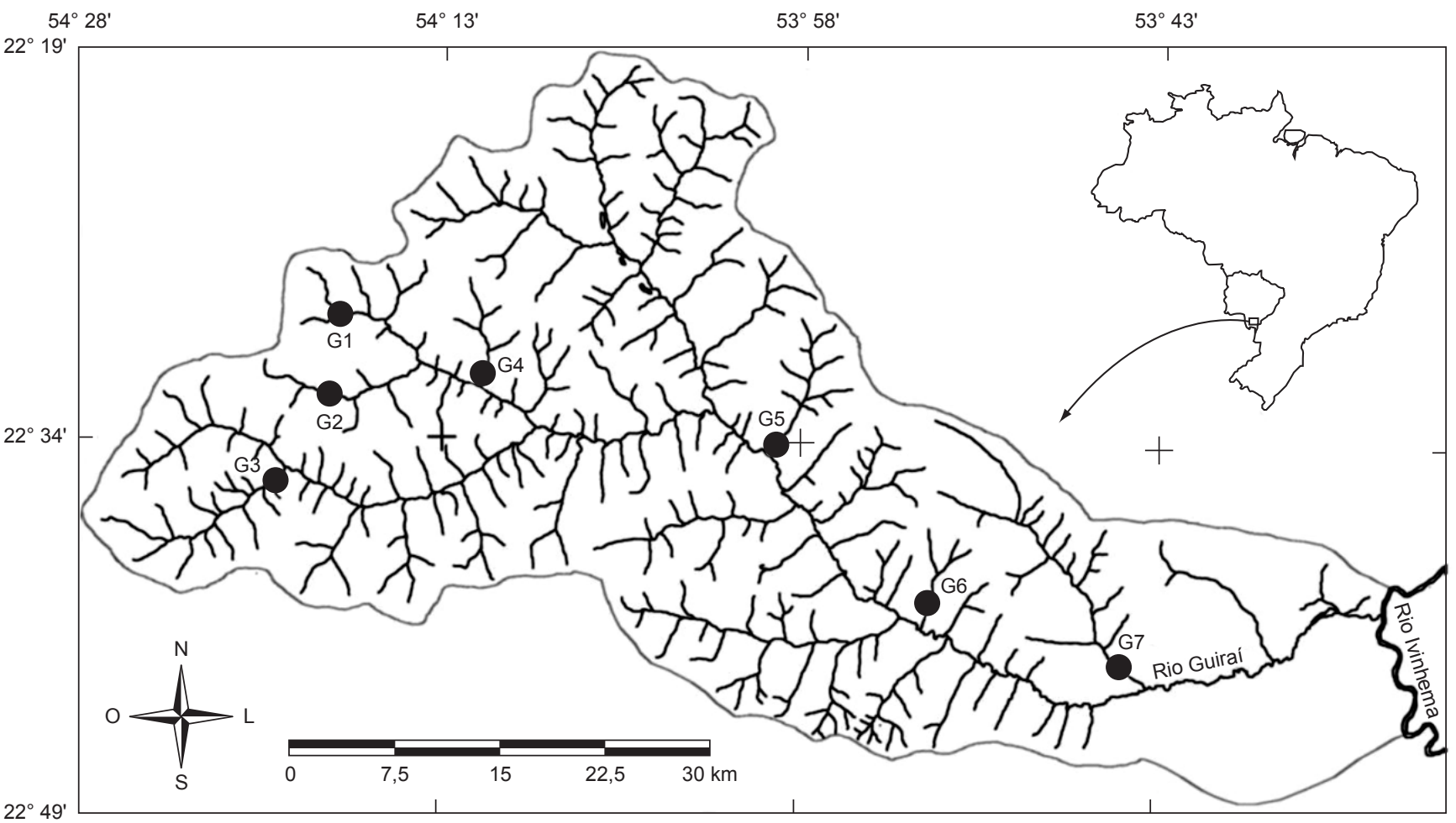

Figura 1. Mapa da bacia do rio Guiraí, Alto Rio Paraná, com os locais amostrados entre outubro/2006 e agosto/2007.

Figure 1. Map of Guiraí river basin, Upper Paraná River, with the locations sampled between october/2006 and august/2007.

$$
\mathrm{AI}_{\mathrm{i}}=\mathrm{F}_{\mathrm{i}} * \mathrm{~V}_{\mathrm{i}} / 100
$$

sendo: $\mathrm{AI}_{\mathrm{i}}$ : Índice de Importância do item i na dieta dos indivíduos da amostra; $F_{i}$ : Frequiência de Ocorrência do item i na amostra; e $V_{i}$ : Índice de Análise Volumétrica do item alimentar i na amostra.

A fim de testar se a dieta variou sazonalmente ou espacialmente quanto à hierarquia dos itens alimentares consumidos, a comparação dos resultados obtidos nas diferentes unidades amostrais foi feita pela aplicação do método proposto por Fritz (1974), segundo o qual os itens alimentares são ranqueados (com base na sua importância relativa) em cada uma das amostras e confrontados pelo coeficiente de comparação de postos de Spearman (utilizando-se nível de significância de 0,05).

Paralelamente à análise da composição da dieta, também foi calculado o Índice de Repleção Estomacal, que é utilizado como um indicativo da atividade alimentar de peixes. Para o cálculo desse índice foi utilizada a seguinte expressão (Hyslop 1980):

$$
\mathrm{IR}=100\left(\mathrm{~W}_{\mathrm{E}} / \mathrm{W}_{\mathrm{T}}\right)
$$

sendo: IR: Índice de Repleção Estomacal; $\mathrm{W}_{\mathrm{E}}$ : massa total do conteúdo estomacal em gramas (com aproximação de $0,01 \mathrm{~g}$ ); e $\mathrm{W}_{\mathrm{T}}$ : massa total do peixe em gramas (com aproximação de $0,01 \mathrm{~g}$ ).

Conforme citado anteriormente, as variações observadas para o Índice de Repleção Estomacal podem estar diretamente relacionadas à intensidade da atividade alimentar dos indivíduos. Dessa forma, os resultados obtidos em cada amostra foram comparados utilizando-se o teste de Kruskal-Wallis, complementado, se necessário, pelo teste a posteriori disponível no pacote estatístico BioEstat 2.0 (Ayres et al. 2000).

\section{Resultados}

Foram capturados 960 exemplares de Bryconamericus stramineus, entre outubro de 2006 e agosto de 2007. As amostragens foram divididas em estação chuvosa para indivíduos coletados nos meses de outubro, dezembro e fevereiro e estação seca para os coletados nos meses de abril, junho e agosto (Tabela 2). Os indivíduos foram pesados (em g, com aproximação de $0,01 \mathrm{~g}$ ) e medidos (em cm, com aproximação de $0,01 \mathrm{~cm}$ ), e 350 estômagos foram analisados.

Bryconamericus stramineus apresentou hábito alimentar insetívoro, consumindo 17 itens alimentares diferentes (Tabela 3). No ponto G1 na estação chuvosa, alimentou-se principalmente de insetos da Ordem Diptera, que ocorreu em 75,76\% dos estômagos com abundância de $26,70 \%$ e 20,23\% de Índice de importância. Na estação seca houve predominância de insetos da Ordem Megaloptera com $50 \%$ de Ocorrência, $15,65 \%$ de abundância e 7,81\% de AI. No ponto G2 a Ordem Hymenoptera ocorreu em 63,93\% dos estômagos com $51,43 \%$ de abundância e AI de 32,88\% na estação chuvosa. Na estação seca a Ordem Diptera prevaleceu com 34,48\% de Ocorrência, 9,48\% de abundância e 3,27\% para o Índice de importância. No ponto G4 Coleoptera ocorreu em 63,01\% dos estômagos com abundância de $60,36 \%$ e 38,03\% de AI na estação chuvosa. Na seca a Ordem Diptera foi a mais consumida esteve presente em 32,26\% dos estômagos, com abundância de $8,06 \%$ e AI de $2,6 \%$. No ponto G5 na estação chuvosa a Ordem Hymenoptera ocorreu em 75,71\% dos estômagos, com $75,36 \%$ de abundância e $57,06 \%$ de AI. Na seca, a Ordem Diptera foi mais freqüente ocasião na qual apareceram em $40 \%$ dos estômagos e foi também mais abundante e com maior IA, $16 \%$ e 6,4\% respectivamente. No local G6 o item predominante foram insetos da Ordem Hymenoptera com ocorrência de 70,59\%, abundância de 

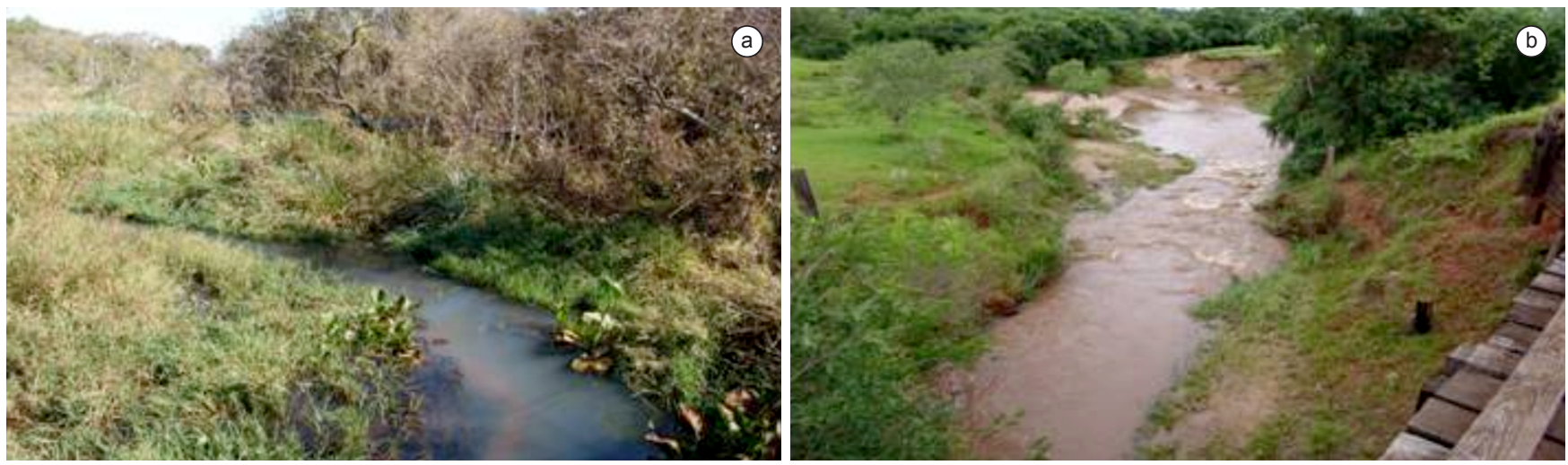

(C)
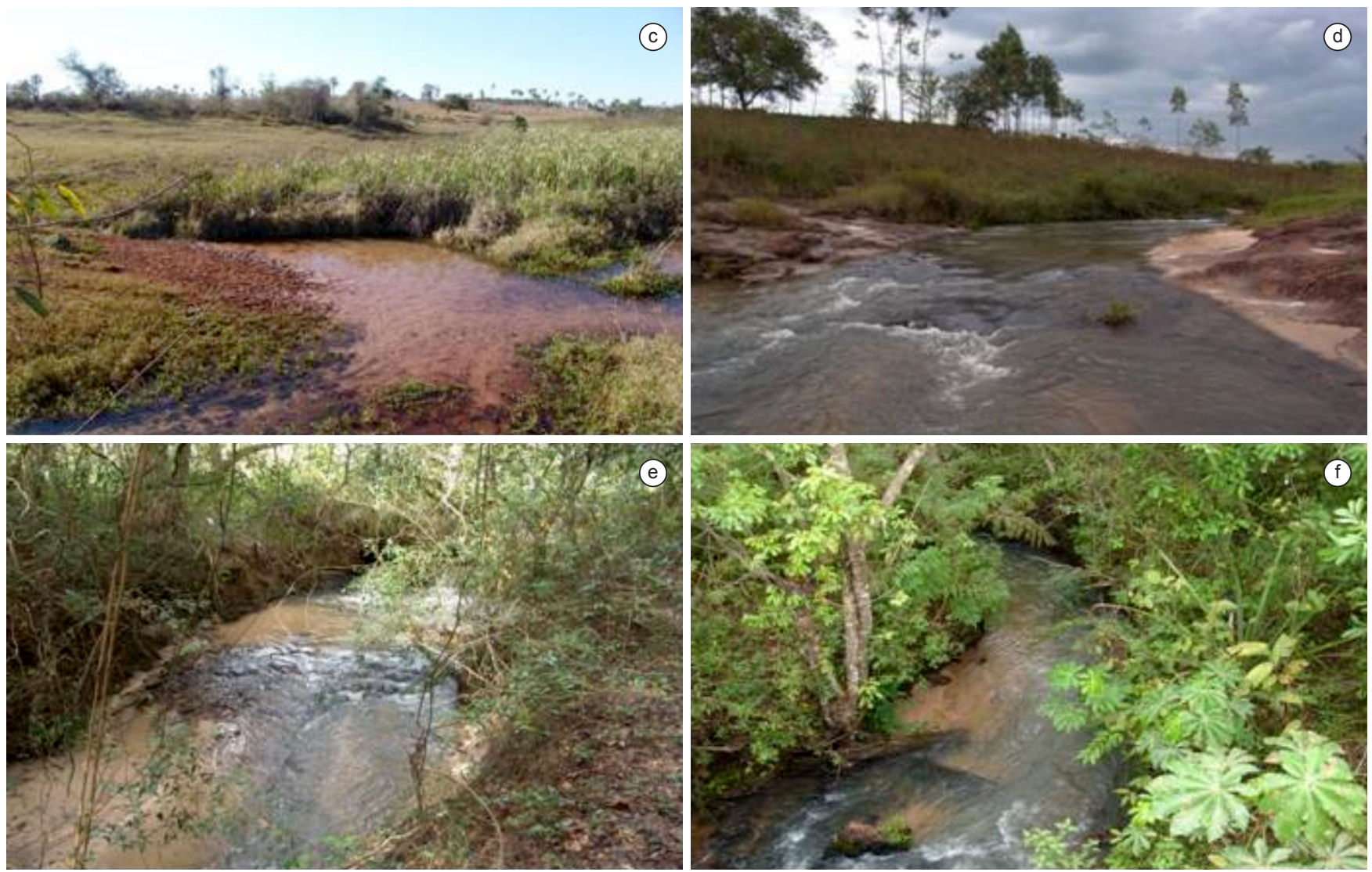

(9)

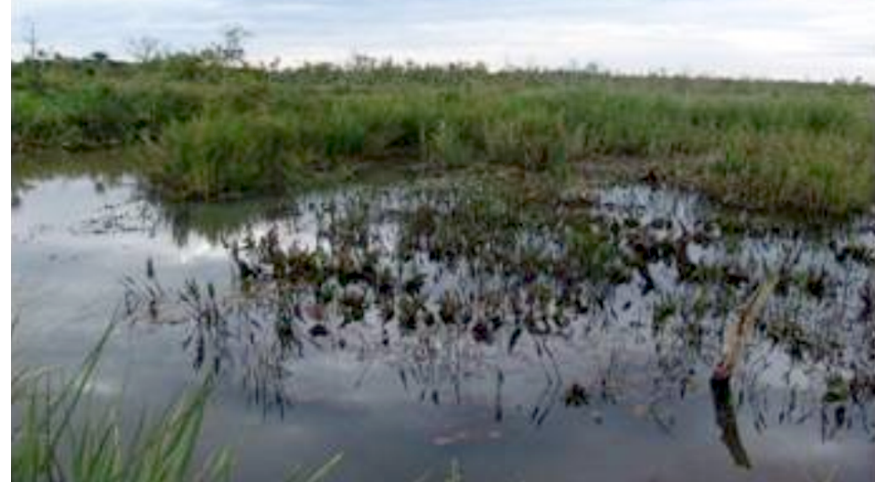

Figura 2. Riachos amostrados na bacia do rio Guiraí, Alto Rio Paraná, a) G1; b) G2; c) G3; d) G4; e) G5; ;f) G6; e h) G7.

Figure 2. Streams sampled in the basin of the river Guiraí, Upper Paraná River, a) G1; b) G2; c) G3; d) G4; e) G5; f) G6; and h) G7. 
Tabela 1. Valores médios e desvio padrão para as variáveis ambientais obtidas nos locais amostrados na bacia do rio Guiraí, Alto Rio Paraná, entre outubro/2006 e agosto/2007.

Table 1. Average values and standard deviation for the environmental variables obtained in the sampled sites in the basin of the River Guiraí, Upper Paraná River, Between October/2006 and August/2007.

\begin{tabular}{|c|c|c|c|c|c|c|c|}
\hline Variáveis/locais & G1 & G2 & G3 & G4 & G5 & G6 & G7 \\
\hline Ordem & $2^{a}$ & $2^{a}$ & $3^{a}$ & $2^{a}$ & $3^{a}$ & $2^{a}$ & $3^{\mathbf{a}}$ \\
\hline \multirow[t]{2}{*}{ Largura (m) } & 1,8 & 5,2 & 1,2 & 4,4 & 4,5 & 3,4 & 6,5 \\
\hline & $\pm 0,1$ & $\pm 0,5$ & $\pm 0,1$ & $\pm 0,2$ & $\pm 0,3$ & $\pm 0,2$ & $\pm 0,4$ \\
\hline \multirow[t]{2}{*}{ Profundidade $(\mathrm{cm})$} & 65 & 73 & 26 & 51 & 51 & 59 & 126 \\
\hline & $\pm 7,3$ & $\pm 15,9$ & $\pm 5,7$ & $\pm 16,5$ & $\pm 5,0$ & $\pm 7,3$ & $\pm 5,3$ \\
\hline \multirow[t]{2}{*}{ Velocidade $(\mathrm{m} / \mathrm{s})$} & 0,4 & 0,6 & 0,8 & 0,7 & 0,7 & 0,9 & 0,3 \\
\hline & $\pm 0,2$ & $\pm 0,15$ & $\pm 0,3$ & $\pm 0,3$ & $\pm 0,2$ & $\pm 1,0$ & $\pm 0,02$ \\
\hline Altitude (m) & 368 & 332 & 353 & 308 & 304 & 271 & 244 \\
\hline \multirow[t]{2}{*}{$\mathrm{pH}$} & 6,7 & 6,9 & 6,7 & 6,8 & 5,5 & 6,7 & 6,6 \\
\hline & $\pm 0,8$ & $\pm 0,4$ & $\pm 0,7$ & $\pm 0,7$ & $\pm 1,3$ & $\pm 0,5$ & $\pm 0,6$ \\
\hline \multirow[t]{2}{*}{ Condutividade $(\mu \mathrm{S} / \mathrm{cm})$} & 234,9 & 401 & 231 & 204,7 & 599,7 & 291 & 238,5 \\
\hline & \pm 534 & \pm 938 & \pm 533 & \pm 461 & \pm 1376 & \pm 668 & \pm 544 \\
\hline \multirow[t]{2}{*}{ Turbidez (NTU) } & 14,4 & 24,5 & 7,5 & 13,1 & 42,0 & 46,9 & 19,6 \\
\hline & \pm 8 & $\pm 23,1$ & $\pm 3,1$ & $\pm 1,5$ & \pm 38 & $\pm 86,4$ & $\pm 10,6$ \\
\hline \multirow[t]{2}{*}{ Temperatura $\left({ }^{\circ} \mathrm{C}\right)$} & 22,9 & 22,4 & 22,8 & 24,6 & 23,8 & 24,3 & 23,8 \\
\hline & $\pm 2,5$ & $\pm 2,0$ & $\pm 2,4$ & $\pm 2,8$ & $\pm 1,3$ & $\pm 2,5$ & $\pm 2,3$ \\
\hline
\end{tabular}

Tabela 2. Total de Indivíduos de Bryconamericus stramineus coletados e analisados, provenientes da sub-bacia do rio Guiraí, (Mato Grosso do Sul - Brasil) nas estações seca e chuvosa.

Table 2. Total Individuals of Bryconamericus stramineus collected and analyzed, from the sub-basin of the river Guiraí (Mato Grosso do Sul - Brazil) in the dry and rainy season.

\begin{tabular}{|c|c|c|c|c|c|c|}
\hline \multirow[t]{3}{*}{ Local } & \multicolumn{4}{|c|}{ Estação } & \multirow{2}{*}{\multicolumn{2}{|c|}{ Total }} \\
\hline & \multicolumn{2}{|c|}{ Chuvosa } & \multicolumn{2}{|c|}{ Seca } & & \\
\hline & Indivíduos & Analisados & Indivíduos & Analisados & Indivíduos & Analisados \\
\hline G1 & 60 & 33 & 36 & 8 & 96 & 41 \\
\hline G2 & 154 & 61 & 101 & 29 & 255 & 90 \\
\hline G3 & 1 & 0 & 0 & 0 & 1 & 0 \\
\hline G4 & 172 & 73 & 93 & 31 & 265 & 104 \\
\hline G5 & 211 & 70 & 77 & 25 & 288 & 95 \\
\hline G6 & 45 & 17 & 10 & 3 & 55 & 20 \\
\hline G7 & 0 & 0 & 0 & 0 & 0 & 0 \\
\hline Total & 643 & 254 & 317 & 96 & 960 & 350 \\
\hline
\end{tabular}

$36,03 \%$ e AI de $25,43 \%$ na estação chuvosa, na estação seca não foram capturados indivíduos suficientes para examinar a dieta (Tabela 2). A análise dos hábitos alimentares evidenciou a grande importância dos insetos como item alimentar (Figura 3).

A análise pelo teste de correlação de Spearman (Tabela 4) indicou que apenas em G1 houve diferença significativa entre as abundâncias de pelo menos dois itens, entre o período chuvoso e o seco, evidenciando uma possível sazonalidade na dieta dos indivíduos desta localidade.

Os índices de repleção no período chuvoso, através da comparação pelo teste de Kruskal-Wallis demonstraram que houve diferença significativa (Tabela 5) na intensidade da atividade alimentar nos indivíduos do Ponto G4 em relação aos demais (G1, G2, G5 e G6). Observando os valores dos postos médios (Figura 4) nota-se uma diferença expressiva entre G4 e o restante dos locais, a diferença entre os índices de repleção pode ser representada pela expressão: $\mathrm{IR}_{\mathrm{G} 6}>\mathrm{IR}_{\mathrm{G} 2}>\mathrm{IR}_{\mathrm{G} 5}>\mathrm{IR}_{\mathrm{G} 1}>\mathrm{IR}_{\mathrm{G} 4}$.
No período seco, as comparações pelo teste de Kruskal-Wallis, evidenciaram uma diferença significativa (Tabela 6) na intensidade alimentar dos espécimes dos pontos G1 e G2 e entre G1 e G5, já entre os demais locais não houve diferença significativa. $\mathrm{O}$ período seco apresentou $\mathrm{IR}_{\mathrm{G} 2}>\mathrm{IR}_{\mathrm{G} 5}>\mathrm{IR}_{\mathrm{G} 4}>\mathrm{IR}_{\mathrm{G} 1}$ (Figura 4).

\section{Discussão}

As espécies pertencentes ao gênero Bryconamericus, nos estudos acerca da alimentação, são descritas como onívoras ou generalistas (Escalante \& Menni1999, Rezende \& Mazzoni 2003), insetívoras (Russo et al. 2004) e detritívoras (Castro 2003).

Bryconamericus stramineus é um caracídeo de pequeno porte, de dieta predominantemente insetívora (Casatti et al. 2003), que coleta itens alimentares transportados pela corrente (Grant \& Noakes 1987), pupas de dípteros associadas a caules de macrófitas e, às vezes, insetos terrestres capturados na superfície (Sazima 1986), além de itens 
Tabela 3. Itens alimentares consumidos por Bryconamericus stramineus nos diferentes pontos de coleta na sub-bacia do rio Guiraí (Mato Grosso do Sul Brasil), nas estações seca e chuvosa.

Table 3. Food items consumed by Bryconamericus stramineus in different points of collection in the sub-basin of the river Guiraí (Mato Grosso do Sul - Brazil), in dry and rainy seasons.

\begin{tabular}{|c|c|c|c|c|c|c|c|c|c|c|}
\hline \multirow[t]{3}{*}{ Item } & \multicolumn{10}{|c|}{ Pontos } \\
\hline & \multicolumn{2}{|c|}{ G1 } & \multicolumn{2}{|c|}{ G2 } & \multicolumn{2}{|c|}{ G4 } & \multicolumn{2}{|c|}{ G5 } & \multicolumn{2}{|c|}{ G6 } \\
\hline & Chuva & Seca & Chuva & Seca & Chuva & Seca & Chuva & Seca & Chuva & Seca \\
\hline Diptera & $\mathrm{x}$ & $\mathrm{x}$ & $\mathrm{x}$ & $\mathrm{x}$ & $\mathrm{x}$ & $\mathrm{x}$ & $\mathrm{x}$ & $\mathrm{x}$ & $\mathrm{x}$ & - \\
\hline Coleoptera & $\mathrm{x}$ & $\mathrm{x}$ & $\mathrm{x}$ & $\mathrm{x}$ & $\mathrm{x}$ & $\mathrm{x}$ & $\mathrm{x}$ & $\mathrm{x}$ & $\mathrm{x}$ & $\mathrm{x}$ \\
\hline Homoptera & $\mathrm{x}$ & $\mathrm{x}$ & $\mathrm{x}$ & $\mathrm{x}$ & $\mathrm{x}$ & $\mathrm{x}$ & - & - & - & - \\
\hline Orthoptera & $\mathrm{x}$ & - & $\mathrm{x}$ & $\mathrm{x}$ & - & $\mathrm{x}$ & $\mathrm{x}$ & $\mathrm{x}$ & - & - \\
\hline Hymenoptera & $\mathrm{x}$ & - & $\mathrm{x}$ & $\mathrm{x}$ & $\mathrm{x}$ & $\mathrm{x}$ & $\mathrm{x}$ & $\mathrm{x}$ & $\mathrm{x}$ & $\mathrm{x}$ \\
\hline Collembola & - & $\mathrm{x}$ & $\mathrm{x}$ & - & - & $\mathrm{x}$ & - & - & - & - \\
\hline Isoptera & $\mathrm{x}$ & $\mathrm{x}$ & - & - & $\mathrm{x}$ & - & - & - & $\mathrm{x}$ & - \\
\hline Megaloptera & - & $\mathrm{x}$ & $\mathrm{x}$ & $\mathrm{x}$ & $\mathrm{x}$ & - & - & - & - & - \\
\hline Ephemeroptera & - & $\mathrm{x}$ & $\mathrm{x}$ & $\mathrm{x}$ & $\mathrm{x}$ & $\mathrm{x}$ & $\mathrm{x}$ & $\mathrm{x}$ & - & - \\
\hline Odonata & $\mathrm{x}$ & - & $\mathrm{x}$ & $\mathrm{x}$ & $\mathrm{x}$ & $\mathrm{x}$ & $\mathrm{x}$ & $\mathrm{x}$ & - & - \\
\hline Thrichoptera & - & $\mathrm{x}$ & $\mathrm{x}$ & $\mathrm{x}$ & $\mathrm{x}$ & $\mathrm{x}$ & $\mathrm{x}$ & $\mathrm{x}$ & - & - \\
\hline Hemiptera & $\mathrm{x}$ & - & $\mathrm{x}$ & $\mathrm{x}$ & $\mathrm{x}$ & - & - & $\mathrm{x}$ & $\mathrm{x}$ & - \\
\hline Plecoptera & $\mathrm{x}$ & - & $\mathrm{x}$ & $\mathrm{x}$ & - & $\mathrm{x}$ & $\mathrm{x}$ & $\mathrm{x}$ & - & - \\
\hline Araneae & - & - & $\mathrm{x}$ & $\mathrm{x}$ & $\mathrm{x}$ & - & - & $\mathrm{x}$ & $\mathrm{x}$ & - \\
\hline Nematoda & - & - & - & - & $\mathrm{x}$ & $\mathrm{x}$ & - & - & - & - \\
\hline Não identificado & $\mathrm{x}$ & $\mathrm{x}$ & $\mathrm{x}$ & $\mathrm{x}$ & $\mathrm{x}$ & $\mathrm{x}$ & $\mathrm{x}$ & $\mathrm{x}$ & $\mathrm{x}$ & $\mathrm{x}$ \\
\hline Sedimento & - & - & - & - & - & - & $\mathrm{x}$ & - & $\mathrm{x}$ & - \\
\hline
\end{tabular}

autóctones, como moluscos, nematóides, insetos aquáticos e algas (Casatti et al. 2003).

O hábito de consumir insetos como principal recurso alimentar é amplamente estabelecido entre espécies de peixes de riachos (Casatti et al. 2003, Casatti et al. 2001, Rezende \& Mazzoni 2003; Russo et al. 2004). Para Lowe-McConnel (1999) insetos requerem de seu predador menor especialização nos hábitos alimentares.

Bryconamericus stramineus, nos riachos da sub-bacia do rio Guiraí, pode ser classificada como uma espécie insetívora, sendo que a tática de consumo das presas variou entre as estações e a as localidades. Durante a estação chuvosa prevaleceram nos estômagos de B. stramineus as ordens de insetos Hymenoptera nos pontos G2, G5 e G6, Diptera no ponto G1 e Coleoptera no ponto G4. Durante a seca predominaram as ordens Megaloptera em G1 e Diptera em G2, G4 e G5. A análise pelo teste de correlação de Spearman sugere sazonalidade na dieta dos indivíduos coletados em G1, pois houve predominância na captura de insetos da Ordem Diptera na estação chuvosa, enquanto que, na seca, houve captura mais intensiva de insetos da Ordem Megaloptera. Efeitos da sazonalidade foram verificados em diferentes comunidades de peixes de riachos (Little et al. 1998, Deus \& Petrere-Junior 2003). Para Oricolli \& Bennemann (2006), as espécies generalistas, cuja flexibilidade trófica é conhecida, mudam de dieta conforme o local e as interações com outras espécies, e sofrem influência da disponibilidade dos recursos ao longo do tempo, o que demonstra bem seu oportunismo, o qual, segundo Gerking (1994), está mais relacionado à súbita abundância de um alimento em uma determinada época. Em alguns casos são detectadas variações na utilização de presas preferenciais de acordo com a disponibilidade do ambiente (Deus \& Petrere-Junior 2003).

Houve diferença significativa na atividade alimentar no período chuvoso, entre o ponto G4 e os demais, em G4 os exemplares de- monstraram uma atividade alimentar baixa em relação aos de outros locais analisados. Os indivíduos em G6, local que apresenta maior cobertura de mata ciliar (Figura 2f), apresentaram um maior índice de repleção, se alimentaram mais intensamente que os indivíduos de G2, G5, G1 e G4, respectivamente. Os itens alimentares autóctones a alóctones são dependentes da mata marginal (Alvim \& Peret 2004) e a conservação destas áreas garante o provimento de grande variedade de itens alimentares para a ictiofauna (Lowe-Mcconnell 1999), podendo ocorrer variações espaço-temporais na disponibilidade destes itens (Abelha et al. 2001).

Muitos predadores encontram suas presas na deriva, na vegetação marginal ou associadas a substratos utilizados tanto para alimentação quanto para reprodução (Gil \& Hart 1999). Dentre os itens que compõem a dieta de $B$. stramineus, os insetos alóctones corresponderam a uma importante parcela das presas consumidas, principalmente na estação chuvosa, na qual também foram registrados os maiores índices de repleção. O período de águas altas é o principal período de alimentação e crescimento e de acúmulo de reservas de gorduras. As águas cheias, invadindo as margens, são enriquecidas com nutrientes devido à rápida decomposição de gramíneas e restos animais, ou à camada humífera da floresta, o que leva a um crescimento excessivo de microorganismos, seguido de grande explosão de macroinvertebrados (insetos, crustáceos, moluscos) usados como alimento pelos peixes (Lowe-McConnel 1999). Em riachos, tanto larvas de insetos quanto adultos são itens alimentares importantes para um grande número de espécies de peixes (Sabino \& Castro 1990, Rezende \& Mazzoni 2003, Casatti et al. 2003).

Em todos os riachos analisados, Bryconamericus stramineus apresentou dieta basicamente composta por insetos, sendo os demais itens consumidos ocasionalmente, como o observado por Casatti et al.(2001) em um riacho no Parque Estadual do Morro do Diabo, 

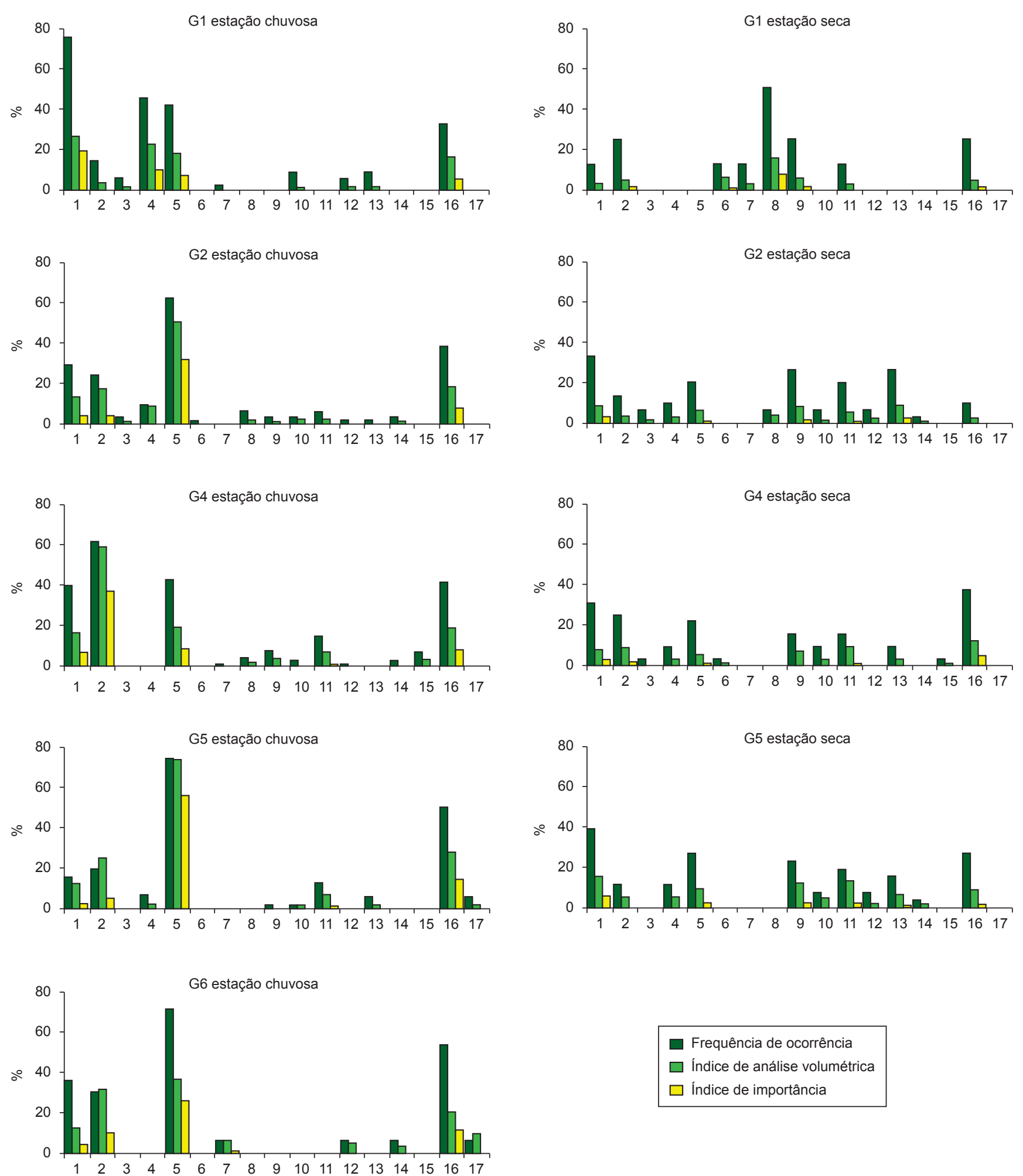

Frequência de ocorrência

$\square$ Índice de análise volumétrica

$\square$ Índice de importância

Figura 3. Itens alimentares observados em estômagos de Bryconamericus stramineus em riachos da sub-bacia do rio Guiraí (1 - Diptera; 2 - Coleoptera; 3 Homoptera; 4 - Orthoptera; 5 - Hymenoptera; 6 - Collembola; 7 - Isoptera; 8 - Megaloptera; 9 - Ephemeroptera; 10 - Odonata; 11 - Trichoptera; 12 - Hemiptera; 13 - Plecoptera; 14 - Araneae; 15 - Nematoda; 16 - Não identificado; e 17 - Sedimento).

Figure 3. Food itens observed in stomachs of Bryconamericus stramineus in streams of Guraí river Sub-Basin (1 - Diptera; 2 - Coleoptera; 3 - Homoptera; 4 Orthoptera; 5 - Hymenoptera; 6 - Collembola; 7 - Isoptera; 8 - Megaloptera; 9 - Ephemeroptera; 10 - Odonata; 11 - Trichoptera; 12 - Hemiptera; 13 - Plecoptera; 14 - Araneae; 15 - Nematoda; 16 - Unidentified; and 17 - Pellet). 
Tabela 4. Análise da variação sazonal da dieta de Bryconamericus stramineus nos diferentes pontos de coleta na sub-bacia do rio Guiraí (Mato Grosso do Sul - Brasil), através do teste de correlação de Spearman ( $p>0,05)$.

Table 4. Analysis of seasonal variation in diet of Bryconamericus stramineus in different points of collection in the sub-basin of the river Guiraí (Mato Grosso do Sul - Brazil) by Spearman's correlation tests ( $\mathrm{p}>0.05)$.

\begin{tabular}{cl}
\hline \multicolumn{2}{c}{ Teste de correlação de Spearman } \\
\hline Pontos & Resultados \\
\hline G1 & $(\mathrm{p})=0,4153$ \\
G2 & $(\mathrm{p})=0,0084$ \\
G4 & $(\mathrm{p})=0,0044$ \\
G5 & $(\mathrm{p})=0,0006$ \\
\hline
\end{tabular}

Tabela 5. Variação da intensidade alimentar de Bryconamericus stramineus nos diferentes pontos de coleta na sub-bacia do rio Guiraí (Mato Grosso do Sul - Brasil), no perído chuvoso através do teste de Kruskal-Wallis $(\mathrm{p}<0,05)$.

Table 5. Change the intensity of food Bryconamericus stramineus in different points of collection in the sub-basin of the river Guiraí (Mato Grosso do Sul Brazil) in the rainy period through the Kruskal-Wallis $(\mathrm{p}<0.05)$.

\begin{tabular}{|c|c|c|c|c|c|}
\hline \multicolumn{6}{|c|}{ Estação chuvosa } \\
\hline \multirow[t]{2}{*}{$H=37,945$} & Gl $=4$ & $p=0,0001$ & & & \\
\hline & G1 & G2 & G4 & G5 & G6 \\
\hline G1 & - & - & - & - & - \\
\hline G2 & ns & - & - & - & - \\
\hline G4 & $<0,05$ & $<0,05$ & - & - & - \\
\hline G5 & ns & ns & $<0,05$ & - & - \\
\hline G6 & ns & ns & $<0,05$ & ns & - \\
\hline
\end{tabular}

Tabela 6. Variação da intensidade alimentar de Bryconamericus stramineus nos diferentes pontos de coleta na sub-bacia do rio Guiraí (Mato Grosso do Sul - Brasil), no perído seco através do teste de Kruskal-Wallis $(\mathrm{p}<0,05)$.

Table 6. Change the intensity of food Bryconamericus stramineus in different points of collection in the sub-basin of the river Guiraí (Mato Grosso do Sul - Brazil) in the dry period through the Kruskal-Wallis $(\mathrm{p}<0.05)$.

\begin{tabular}{ccccc}
\hline \multicolumn{5}{c}{ Estação seca } \\
\hline H = 16,7025 & Gl = 3 & $\mathbf{p}=\mathbf{0 , 0 0 0 8}$ \\
\cline { 2 - 6 } & G1 & $\mathbf{G 2}$ & $\mathbf{G 4}$ & $\mathbf{G 5}$ \\
\hline G1 & - & - & - & - \\
G2 & $<0,05$ & - & - & - \\
G4 & ns & ns & - & - \\
G5 & $<0,05$ & ns & ns & - \\
\hline
\end{tabular}

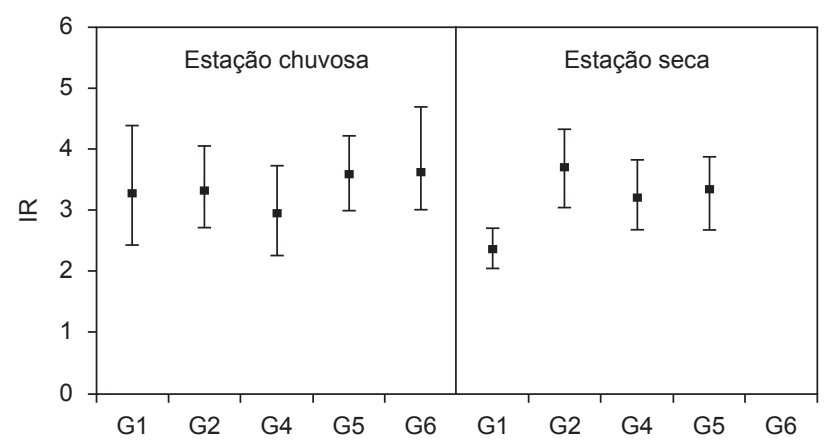

Figura 4. Índice de repleção estomacal por estação e em cada local de amostragem.

Figure 4. Repletion indexes of stomach per season and each sampling locality. corroborando os dados registrados na literatura para outra espécie do mesmo gênero (Rezende \& Mazzoni 2003, para B. microcephalus).

\section{Agradecimentos}

À Fundect (Processo n $\left.{ }^{\circ} 41 / 100.143 / 2006\right)$ pelo financiamento do projeto e à prefeitura municipal de Novo Horizonte do Sul juntamente com Flávio Raphael Matos pelo apoio logístico às atividades de campo. A João Paulo da Silva, Lílian P. Vasconcelos, Marcelo Maldonado de Souza, Sabrina B. Valério, Suelen Ap. de Oliveira e Viviane V. Azevedo pelo auxílio nos trabalhos de campo.

\section{Referências Bibliográficas}

ABELHA, M.C.F., AGOSTINHO, A.A. \& GOULART, E. 2001. Plasticidade trófica em peixes de água doce. Acta Sci. 23(2):425-434.

ALVIM, M.C.C. \& PERET, A.C. 2004. Food resources sustaining the fish fauna in a section of the upper São Francisco River in Três Marias, MG, Brazil. Braz. J. Biol. 64(2):195-202.

AYRES, M., Jr, A. M., LIMA AYRES, D., SANTOS, A. S. DOS. 2000. BioEstat 2.0: aplicações estatísticas nas áreas das ciências biológicas e médicas. Sociedade Civil Mamirauá, Belém. 259 p

BARNES, R.D. 1990. Zoologia dos invertebrados. Roca, São Paulo. 1179p.

CASATTI, L., LANGEANI, F. \& CASTRO, R.M.C. 2001. Peixes de riacho do Parque Estadual Morro do Diabo, bacia do alto rio Paraná, SP. Biota Neotrop. 1(1): http://www.biotaneotropica.org.br/v1n12/pt/abstract?inv entory+BN00201122001 (último acesso em 08/10/2008).

CASATTI, L., MENDES, H.F. \& FERREIRA, K.M. 2003. Aquatic macrophytes as feeding site for small fishes in the Rosana reservoir, Paranapanema river, southeastern Brazil. Braz. J. Biol. 63(2):213-222.

CASTRO, R.J. 2003. Regime alimentar e ecomorfologia das espécies de peixes dominantes de um afluente do reservatório de Jurumirim (alto rio Paranapanema, São Paulo). Tese de Doutorado, Universidade Estadual Paulista, Botucatu.

DEUS, C.P. \& PETRERE-JUNIOR, M. 2003. Seasonal diet shifts on seven fish species in an Atlantic Rainforest stream in southeastern Brazil. Braz. J. Biol. 63(4):579-588.

ESCALANTE, A.H. \& MENNI, R.C. 1999. Feeding ecology of the fish Gymnocharacinus bergi, a characid from southern South America. Water SA, 25(4):529-532.

FRITZ, E.S. 1974. Total diet comparison in fishes by Spearman rank correlation coefficients. Copeia, 1:210-214.

GERKING, S.D. 1994. Feeding ecology of fishes. Academic Press, New York. $416 \mathrm{p}$.

GIL, A.B. \& HART, P.J.B. 1999. Dynamic changes in prey choice by stickleback during simultaneous encounter with large prey. J. Fish Biol. 55(6): 1317-1327.

GRANT, J.W.A. \& NOAKES, D.L.G. 1987. A simple model of optimal territory size for drift- feeding fishes. Can. J. Zool. 65:270- 276.

HAHN, N.S., AGOSTINHO, A.A. \& GOITEIN, R. 1997. Feeding ecology of curvina Plagioscion squamosissimus (Heckel, 1840) (Osteichthyes, Perciformes) in the Itaipu Reservoir and Porto Rico floodplain. Acta Limnol. Bras. 9:11-22.

HYSLOP, E.J. 1980. Stomach content analysis: a review of methods and their applications. J. Fish Biol. 17:411-429.

LIMA-JUNIOR, S.E. \& GOITEIN, R. 2001. A new method for the analysis of fish stomach contents. Acta Sci. 23:421-424.

LIMA-JUNIOR, S.E. 2004. A ictiofauna e a qualidade da água em trechos do rio Corumbataí - SP. Tese de Doutorado, Instituto de Biociências, Universidade Estadual Paulista, Rio Claro. 232f.

LITTLE, A.S., TONN, W.M., TALLMAN, R.F. \& REIST, J.D. 1998. Seasonal variation in diet and trophic relationships within the fish communities of the lower Slaver River, Northwest Territories, Canada. Environ. Biol. Fish, 53:429-445. 
LOWE-McCONNELL, R.H. 1999. Estudos ecológicos de comunidade de peixes tropicais. Editora da Universidade de São Paulo, São Paulo. 535p.

NEEDHAM, J.G. \& NEEDHAM, P.R. 1982. Guia para el estudio de los seres vivos de las aguas dulces. Reverté, Barcelona. 131p.

ORICOLLI, M.C.G., BENNEMANN, S.T. 2006. Dieta de Bryconamericus iheringii (Ostariophysi: Characidae) em riachos da bacia do rio Tibagi, Estado do Paraná. Acta Sci. Biol. Sci. 28(1):59-63.

PETERSON, A. 1960. Larvae of insects: an introduction to Neartic Species (Part II). Ohio State University, Ohio. 416p.

REZENDE, C.F., MAZZONI, R. 2003. Aspectos da alimentação de Bryconamericus microcephalus (Characiformes, Tetragonopterinae) no córrego Andorinha, Ilha Grande, RJ. Biota Neotrop. (3)1: $<\mathrm{http}: / /$ www.biotaneotropica.org.br/v3n1/pt/abstract?shortcommunication+BN01603012003> (último acesso em 08/10/2008).

ROSS, S.T. 1986. Resource partitioning in fish assemblages: a review of field studies. Copeia, 2:352-388.
RUSSO, M.R., HAHN, N.S., PAVANELLI, C.S. 2004. Resource partitioning between two species of Bryconamericus Eigenmann, 1907 from the Iguaçu river basin, Brazil. Acta Biol. Sci. 26(4):431-436.

SABINO, J. \& CASTRO, M.C. 1990. Alimentação, período de atividade e distribuição espacial dos peixes de um riacho da Floresta Atlântica (sudeste do Brasil). Rev. Bras. Biol. 50(1):23-36.

SAZIMA, I. 1986. Similarities in feeding behavior between some marine and freshwater fishes in two tropical communities. J. Fish. Biol. 29(1):53-65.

WOOTTON, R.J. 1992. Fish Ecology. Ed. Chapman \& Hall, New York. 212p.

ZAVALA-CAMIN, L.A. 1996. Introdução aos estudos sobre alimentação natural em peixes. Eduem/Nupelia, Maringá. 129p.

Recebido em 21/10/08 Versão reformulada recebida em 15/03/09

Publicado em 30/03/09 
